Original Article

\title{
Clinical frailty adds to acute illness severity in predicting mortality in hospitalized older adults: An observational study弦
}

\author{
Roman Romero-Ortuno a,b,*, Stephen Wallis a , Richard Biram a , Victoria Keevil ${ }^{\text {a,b }}$ \\ a Department of Medicine for the Elderly, Addenbrooke's Hospital, Cambridge, United Kingdom \\ b Clinical Gerontology Unit, Department of Public Health and Primary Care, University of Cambridge, United Kingdom
}

\section{A R T I C L E I N F O}

\section{Article history:}

Received 9 June 2016

Received in revised form 9 August 2016

Accepted 29 August 2016

Available online 2 September 2016

\section{Keywords:}

Frail elderly

Hospital medicine

Mortality

Patient acuity

Survival analysis

\begin{abstract}
A B S T R A C T
Aim: Frail individuals may be at higher risk of death from a given acute illness severity (AIS), but this relationship has not been studied in an English National Health Service (NHS) acute hospital setting.

Methods: This was a retrospective observational study in a large university NHS hospital in England. We analyzed all first non-elective inpatient episodes of people aged $\geq 75$ years (all specialties) between October 2014 and October 2015. Pre-admission frailty was assessed with the Clinical Frailty Scale (CFS) of the Canadian Study on Health \& Aging, and AIS in the Emergency Department was measured with a Modified Early Warning Score (ED-MEWS $<4$ was considered as low acuity, and ED-MEWS $\geq 4$ as high acuity). A survival analysis compared times to 30-day inpatient death between CFS categories (1-4: very fit to vulnerable, 5: mildly frail, 6: moderately frail, and 7-8: severely or very severely frail).

Results: There were 12,282 non-elective patient episodes ( 8202 first episodes, of which complete data was available for 5505). In a Cox proportional hazards model controlling for age, gender, Charlson Comorbidity Index, history of dementia, current cognitive concern, and discharging specialty (medical versus surgical), ED-MEWS $\geq 4$ $(\mathrm{HR}=2.87,95 \% \mathrm{CI}: 2.27-3.62, \mathrm{p}<0.001)$, and CFS 7-8 (compared to CFS $1-4, \mathrm{HR}=2.10,95 \% \mathrm{CI}: 1.52-2.92$, $\mathrm{p}<0.001$ ) were independent predictors of survival time.

Conclusions: We found frailty and AIS independently associated with inpatient mortality after adjustment for confounders. Hospitals may find it informative to undertake large scale assessment of frailty (vulnerability), as well as AIS (stressor), in older patients admitted to hospital as emergencies.
\end{abstract}

(c) 2016 European Federation of Internal Medicine. Published by Elsevier B.V. All rights reserved.

\section{Introduction}

Over the last decade widespread efforts within the English National Health Service (NHS) have been made to improve the early detection of patients at risk of adverse outcomes. In acute healthcare settings, such as Emergency Departments (EDs), patients have acute illness severity (AIS) information collected on arrival. One way of measuring AIS is by considering the degree of derangement of routinely collected physiological parameters, incorporated into an early warning score (EWS) [1]. Early warning scores grade the risk of patient deterioration and can help guide healthcare staff to escalate clinical care according to pre-specified protocols $[2,3]$. However, it has been suggested that AIS

\footnotetext{
th Authors' contributions: Roman Romero-Ortuno conceived the study, collected and interpreted data, performed statistical analyses, and prepared the manuscript. Stephen Wallis and Richard Biram collected and interpreted data and revised the manuscript critically for important intellectual content. Victoria Keevil performed statistical analyses, interpreted data, and revised the manuscript critically for important intellectual content. All authors read and approved the final manuscript before submission.

* Corresponding author at: Department of Medicine for the Elderly, Box 135, Addenbrooke's Hospital, Hills Road, Cambridge CB2 0QQ United Kingdom.

E-mail address: roman.romero-ortuno@nhs.net (R. Romero-Ortuno).
}

may not be the only driver of poor hospital outcomes, especially in those with a high burden of chronic disabling disease [4], and the proportion of older people admitted to NHS acute hospitals across England with pre-existing syndromes of multi-morbidity, cognitive impairment, mobility problems and physical dependency has increased dramatically over recent years [5].

Those syndromes can be identified early during the admission and may confer vulnerability to adverse outcomes due to reduced physiological reserve and ability to withstand acute stressors, a concept encapsulated by frailty. Frailty in older adults has been defined as a state of vulnerability due to cumulative decline in many physiological systems, which depletes homoeostatic reserves and results in poor restoration of homoeostasis after a stressor event triggering disproportionate changes in health status [6]. It is thought that physiological vulnerability in older adults is gradable along a continuum between fitness (or resilience) and frailty, and chronological age alone cannot accurately tell where a person is along that spectrum [7]. Indeed, the relationship between chronological age and health status is very variable [8]. However, unlike AIS, frailty is not routinely measured in acute healthcare settings.

While the concept of frailty as an age-independent, gradable state of vulnerability to poor outcomes from stressors is quite uncontroversial 
[6], a number of approaches exist to its operationalization in clinical practice [9]. It is increasingly recognized that a gold standard approach for measuring frailty is neither appropriate nor desirable, and that different validated instruments can be suited to different settings and/or purposes $[10,11]$. In the NHS, the assessment of frailty in the acute, inpatient setting could add value to the management of the growing -but heterogeneouspopulation of older people [12-14]. Therefore, the measurement of frailty in acute settings is being encouraged by national initiatives such as the Acute Frailty Network (http://www.acutefrailtynetwork.org.uk). However, practitioners still report several major barriers preventing frailty from being rapidly measured at the front door [15], and there are still no national incentives to remove some of those barriers.

We utilized routinely collected data from a large tertiary university hospital in England where both AIS (proxy for acute stressor) and frailty (proxy for baseline vulnerability) are routinely measured on admission. We hypothesized that these two different entities may independently impact upon the risk of death in acute older adults. Previous research studies with frailty scores in acute settings have not been able to simultaneously consider AIS [16], and our aim was to study the relation between frailty, acuity and mortality in a real-world NHS setting.

\section{Methods}

\subsection{Setting}

This retrospective observational study was conducted in a large tertiary university hospital in England with 1000 acute beds receiving over 102,000 visits to the ED and admitting over 73,000 patients per year; among the latter, over 12,000 are aged 75 or more years.

\subsection{Sample}

We analyzed all first non-elective inpatient episodes (i.e. from ED admission to discharge) of people aged $\geq 75$ years (all specialties) between the 26th of October 2014 and the 26th of October 2015. Data was obtained via the hospital's information systems following the implementation of a new electronic patient record (eHospital system) on the 26th of October 2014.

\subsubsection{Patients' characteristics and outcomes}

The following variables were extracted from the hospital's information systems:

- Age and gender.

- Discharge specialty (medical versus surgical).

- Charlson Comorbidity Index (CCI, without age adjustment) [17]. The $\mathrm{CCI}$ is based on the discharge diagnoses, as coded by the 10th version of the WHO International Classification of Diseases (ICD-10). Therefore, it was calculated retrospectively and would have not been available to clinicians early during the patients' admission.

- Frailty. A frailty instrument that evaluates pre-admission comorbidity, cognitive impairment and disability is the Clinical Frailty Scale (CFS) of the Canadian Study on Health \& Aging (http://geriatricresearch. medicine.dal.ca/clinical_frailty_scale.htm) [18]. The use of the CFS has been found to be feasible in real-world acute NHS settings [19]. The use of the CFS in admissions of people aged $\geq 75$ years was introduced in our center in 2013 under a local Commissioning for Quality and Innovation (CQUIN) scheme (http://www.institute.nhs.uk/ commissioning/pct_portal/cquin.html) [20]. The CQUIN required that all patients aged 75 years or over admitted to the hospital, via the emergency pathway, be screened for frailty using the CFS within $72 \mathrm{~h}$ of admission. The new electronic patient admission screen includes a CFS scoring section as per http://geriatricresearch.medicine. dal.ca/clinical_frailty_scale.htm. The admitting doctor usually scores the CFS, but it can also be completed by ED nurses or by nursing or therapy staff from the trust-wide Specialist Advice for the Frail Elderly
(SAFE) team. Training on CFS scoring is provided to staff on induction and at regular educational meetings. In order to avoid confounding, patients with a CFS of 9 ('terminally ill' without being evidently frail) were excluded from the analyses. To avoid statistical underpower (due to a relatively low number of deaths), the original CFS categories were collapsed into four ordinal categories: up to vulnerable (CFS 1 to 4), mildly frail (CFS 5), moderately frail (CFS 6), and severely or very severely frail (CFS 7 or 8 ).

- AIS information is routinely collected by the nurses in the ED immediately after presentation (i.e. during the patient's triage) and throughout the patient's time in the ED (less than $4 \mathrm{~h}$ in the majority of cases) using a Modified Early Warning Score (ED-MEWS). The components and scoring of the ED-MEWS are shown in Appendix A. Where more than one ED-MEWS was collected during the patient's time in the ED, the highest was used in the analyses. In our ED, the usual ED-MEWS trigger for escalation (i.e. request for immediate medical review) is 4 or more points. Thus, ED-MEWS $\geq 4$ defined high acuity in our analyses.

- Known history of dementia without a current cognitive concern (identified as 'yes' in the database). This was also collected by the admitting team within the first $72 \mathrm{~h}$ of the admission in patients aged 75 or more, thanks to a parallel local CQUIN scheme. An additional variable was collected reflecting current cognitive concern without history of dementia (yes versus no).

- Length of stay (LOS, days).

- Inpatient mortality up to 30 days since admission (\%). The rationale for the 30-day cut-off was to avoid capturing the deaths of long-stay patients, which could be less related to initial AIS. Information on detailed causes of death was not available on the service evaluation database.

\subsection{Statistical analyses}

All statistics were computed with IBM $®$ SPSS $®$ Statistics Version 22. The bivariate correlation between the ED-MEWS (continuous score) and the CFS categories was assessed with the two-sided Spearman's rho correlation coefficient. Other bivariate comparisons were conducted with the non-parametric independent-samples Mann-Whitney U test (continuous versus dichotomous variables) or the Chi-squared test (between categorical variables), as appropriate. Ninety-five percent confidence intervals (CI) for 30-day inpatient mortality were computed.

Age and sex adjusted survival curves were calculated on the overall sample to compare times to 30-day inpatient death across CFS categories. A Cox proportional hazards regression model was used to test the independent effects of frailty and acuity in predicting time to death while adjusting for age, gender, discharging specialty (medical vs. surgical), CCI, history of dementia, and current cognitive concern. Hazard Ratios (HR) with 95\% CI were calculated for the predictors. To check the classification ability of the model, we saved the individuallevel cumulative hazard function and we plotted it against 30-day inpatient mortality in an area under the curve (AUC) analysis. To check the proportional hazards (PH) assumption for the covariates, we plotted the cumulative hazards functions for the covariates, categorizing the continuous ones. Covariates were considered to fulfill the $\mathrm{PH}$ assumption if lines did not cross each other on the plots.

As a sensitivity analysis, the Cox proportional hazards regression analysis was repeated after imputing missing CFS and ED-MEWS scores using multiple imputation by chained equations.

\subsection{Ethics approval}

This Service Evaluation Audit was registered with our center's Safety and Quality Support Department (Project register number 3962). 
Formal confirmation was received that approval from the Ethics Committee was not required.

\subsection{Declaration of sources of funding}

This service evaluation did not receive any specific grant from funding agencies in the public, commercial, or not-for-profit sectors.

\section{Results}

The initial database contained 12,282 non-elective admission and discharge episodes of patients aged 75 or more years between the 26th of October 2014 and the 26th of October 2015 (all specialties). Among those, 8202 (66.8\%) were first episodes.

Among the 8202 first episodes, the mean age was 84.1 years (range: 75 to 105 , SD 5.9), $56.5 \%$ were women, and $72.1 \%$ were discharged by a medical specialty. The median CCI was 2 (range: $0-23$ ), 10.0\% had history of dementia without current cognitive concern, and $6.3 \%$ had a current cognitive concern in the absence of known dementia.

Among the 8202 first episodes, the distribution of CFS categories was as follows: 2600 (31.7\%) were up to vulnerable (CFS 1 to 4 ); 1021 (12.5\%) mildly frail (CFS 5); 1324 (16.1\%) moderately frail (CFS 6); $905(11.0 \%)$ severely or very severely frail (CFS 7 or 8); 49 patients (0.6\%) had a CFS of 9 and were excluded from the analyses, and 2303 (28.1\%) had missing CFS data. In terms of initial AIS, 2325 patients (28.4\%) had an ED-MEWS of 4 or more points; 5187 (63.2\%) had an ED-MEWS of less than 4; and 690 (8.4\%) had missing ED-MEWS data. The overall median LOS was 5 days (range: 0-209), and the 30-day inpatient mortality proportion was $6.7 \%$ (548 deaths).

The bivariate correlation between the CFS (continuous scale excluding category 9) and the ED-MEWS (continuous scale) was statistically significant, with a two-tailed Spearman's rho coefficient of 0.17 ( $\mathrm{p}<0.001, \mathrm{n}=5505$ with information for both variables, see Appendix B). Fig. 1 shows the association between the CFS categories and 30-day inpatient mortality, stratified by acuity (310 deaths).

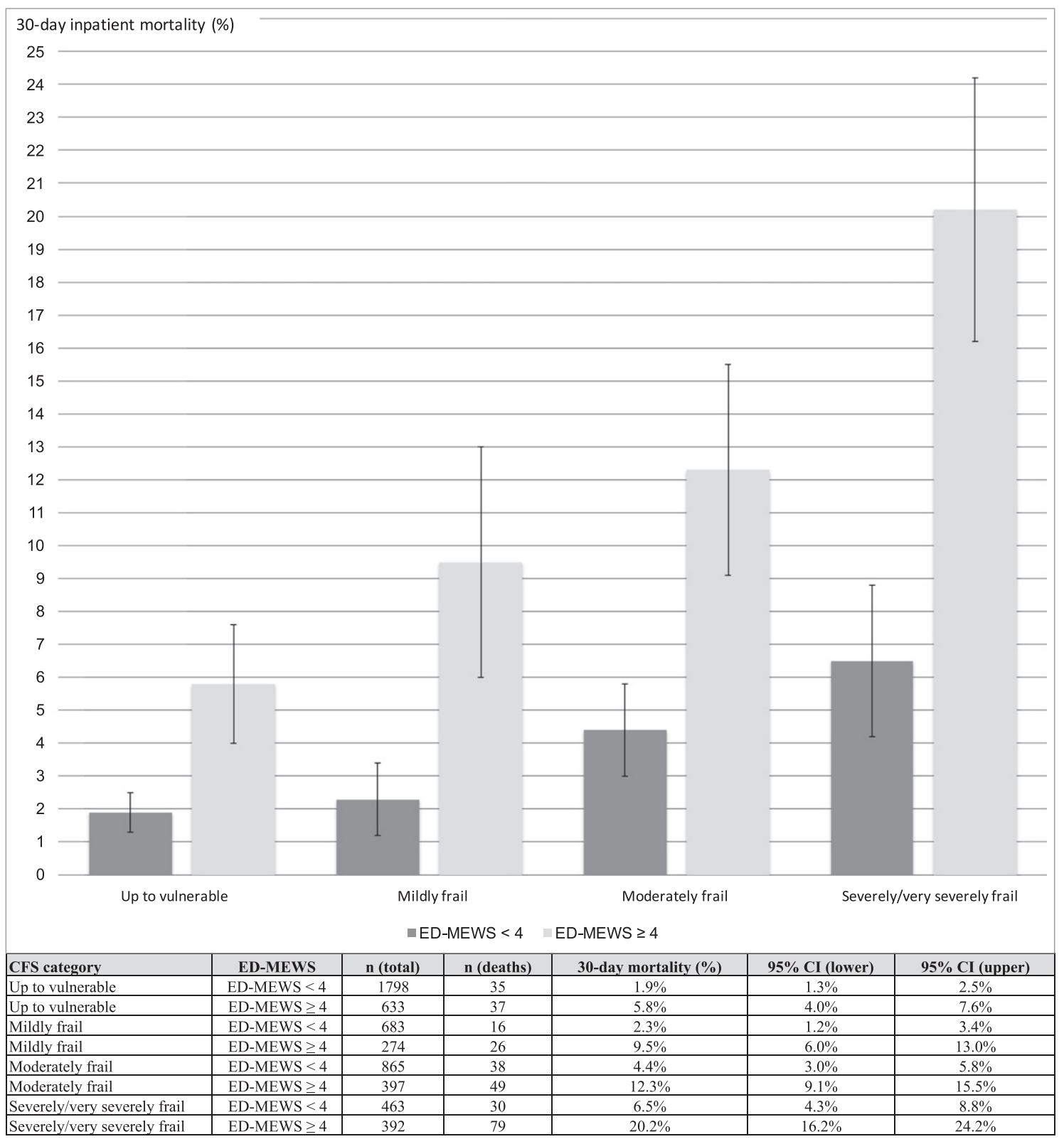

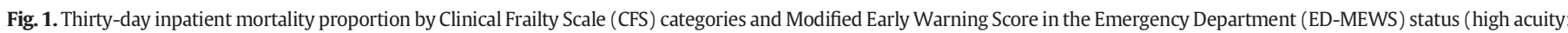
ED-MEWS $\geq 4$; low acuity: ED-MEWS $<4$ ). CI: confidence interval; n: number. 
Two-hundred and thirty-eight deaths could not be included in this analysis due to missing data for either CFS or ED-MEWS. Table 1 shows the characteristics of those included in the analysis $(\mathrm{n}=$ 5505), compared to those with missing information for either CFS or ED-MEWS ( $\mathrm{n}=2697)$.

In the Kaplan-Meier analysis ( $\mathrm{n}=5505)$, the median 30-day survival times for the CFS categories were as follows: up to vulnerable: 27.1 days (95\% CI: 26.4-27.7); mildly frail: 26.3 days (95\% CI: 25.2-27.4); moderately frail: 26.5 days (95\% CI: $25.8-27.2)$; and severely or very severely frail: 24.3 days (95\% CI: 23.3-25.2). Judging by the lack of overlap of $95 \%$ confidence intervals, the survival of the severely or very severely frail stood out as being different from the other three CFS categories. This was visually confirmed by the age and sex adjusted survival curves, which are shown in Fig. 2.

To investigate if acuity and frailty were independent predictors of 30-day inpatient survival time while controlling for potential confounders, a Cox proportional hazards regression model was computed entering the following predictors: ED-MEWS $\geq 4$ (no $=0$, yes $=1$ ), CFS categories (as an ordinal variable, with CFS 1-4 as the reference category), age (as a continuous variable), gender ( male $=0$, female $=1$ ), discharge specialty ( surgical $=0$, medical $=1$ ), $\mathrm{CCl}$ (continuous variable), history of dementia (no $=0$, yes $=1$ ), and current cognitive concern (no $=0$, yes $=1$ ). The result of this model is shown in Table 2 . High acuity and CFS 7-8 were significant independent predictors of survival time. The AUC of the individual-level cumulative hazard function against 30-day inpatient mortality was 0.74 (95\% CI: 0.71-0.76, $\mathrm{p}<0.001$ ), suggesting acceptable discrimination [21]. The plots in Appendix $C$ show the cumulative hazards functions for the covariates in the model, suggesting that the PH assumption was met.

After missing CFS and ED-MEWS scores had been imputed using multiple imputation by chained equations, high acuity, CFS 6 and CFS 7-8 were significant independent predictors of inpatient mortality (see Appendix D).

\section{Discussion}

To our knowledge, this study was the first to test the association between a measure of clinical frailty (vulnerability) and a measure of AIS (stressor) in predicting mortality of hospitalized older adults, in the real-world English National Health Service. Our results suggest that frailty (especially if severe or very severe) adds to acuity in the prediction of inpatient mortality in those aged 75 years or more, independently of potential confounders. It may be helpful to mainstream the measurement of frailty in acute settings in order to enable a more precise patient risk profiling at the front door; in turn, this may help form a basis for targeted interventions and more personalized care pathways [22]. For example, the identification (early during admission) of an adult aged 75 or more years with a CFS of 7 or 8 and an ED-MEWS of 4 or more, signals a high risk of inpatient mortality (20\%), which could then trigger early attempts to personalize the care plan with particular attention to escalation decisions.
In keeping with our results, a previous study of all emergency admissions to an acute Irish hospital over a 12-year period showed that overall, AIS was the best independent predictor of mortality, but chronic disabling disease was an independent predictor of mortality in patients with four or more disabling conditions (i.e. the ones likely to be the frailest) [23]. While chronic disabling disease relies on discharge ICD-10 codes, the CFS can be measured early during the admission, based on a clinical assessment of patients' comorbidity symptoms, and their level of physical activity and dependency on activities of daily living. Thus, the CFS is not only suitable for retrospective observational studies, but also for real life, prospective use.

In our study, the CFS and the ED-MEWS seemed to be two different entities because, although their correlation was statistically significant, the effect size of their correlation was small (Spearman's rho coefficient of 0.17) [24]. A previous study in NHS acute medical units reported a Spearman's rho coefficient of 0.23 between the CFS ( 1 to 9 ) and the National Early Warning Score (NEWS), in keeping with our result [19]. It has been said that frail patients may be 'sicker' in the acute setting because they present later to hospital (lead-time bias) or indeed, their ability to compensate for physiological derangement is impaired [19]. Attempts are often made to manage very frail people in the community, but very acute physiology can become unmanageable in the community without the appropriate resources. For example, care home patients are among the frailest in the community and are often referred to hospital with high acute illness severity [25].

Our study has important limitations, including its single center perspective and the relatively small number of deaths despite a large initial sample size, a problem that was significantly aggravated by missing data. The overall 30-day inpatient mortality proportion was 6.7\% (548 deaths), which is comparable to overall inpatient mortality rates in England for this older age group [26,27]. However, due to our proportion of missing data, we were unable to study 238 out of 548 deaths in the database, with consequent underpower.

As suggested by results in Table 1, those with missing data were younger, more likely to be male, surgical, and to die, and less likely to be cognitively impaired. In that light, it is possible that the association between acuity and frailty and inpatient mortality might be stronger than we reported, since our analyses truncated both the least and most vulnerable (those most likely to be discharged quickly and those most likely to die). This hypothesis is supported by the results of the sensitivity analyses, conducted after multiple imputation of missing CFS and ED-MEWS scores. When Cox proportional hazards regression was repeated in the whole patient cohort, the independent associations between both CFS and ED-MEWS score and inpatient mortality were strengthened (Appendix D).

Although $28 \%$ of our patients had missing CFS scores, we do not think that this necessarily reflects negatively on the feasibility of the CFS in the acute hospital setting. The local CQUIN scheme that mandated measurement of frailty in all older patients admitted via the emergency pathway specified that frailty had to be measured within the first $72 \mathrm{~h}$.

Table 1

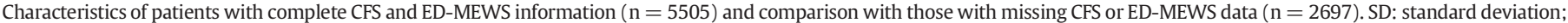
IQR: interquartile range; LOS: length of stay; $†$ Independent-samples Mann-Whitney U test; $\ddagger$ Chi-squared test.

\begin{tabular}{|c|c|c|c|}
\hline & CFS and ED-MEWS not missing $(\mathrm{n}=5505)$ & Missing CFS or ED-MEWS $(\mathrm{n}=2697)$ & $\mathrm{p}$ for difference \\
\hline Mean age, years (SD) & $84.5(5.9)$ & $83.3(5.9)$ & $<0.001 \dagger$ \\
\hline Female gender $(\%)$ & 58.1 & 53.2 & $<0.001 \ddagger$ \\
\hline Discharged by medical specialty (\%) & 78.7 & 58.5 & $<0.001 \ddagger$ \\
\hline Median CCI (IQR) & $2(4)$ & $2(4)$ & $0.505 \dagger$ \\
\hline History of dementia (\%) & 11.4 & 6.9 & $<0.001 \ddagger$ \\
\hline Current cognitive concern (\%) & 7.0 & 4.9 & $<0.001 \ddagger$ \\
\hline Median LOS, days (IQR) & $6(11)$ & $2(8)$ & $<0.001 \dagger$ \\
\hline 30-day inpatient mortality \% (n) & $5.6(310)$ & $8.8(238)$ & $<0.001 \ddagger$ \\
\hline Missing CFS \% (n) & - & $87.2(2352)$ & - \\
\hline Missing ED-MEWS \% (n) & - & $25.6(690)$ & - \\
\hline
\end{tabular}




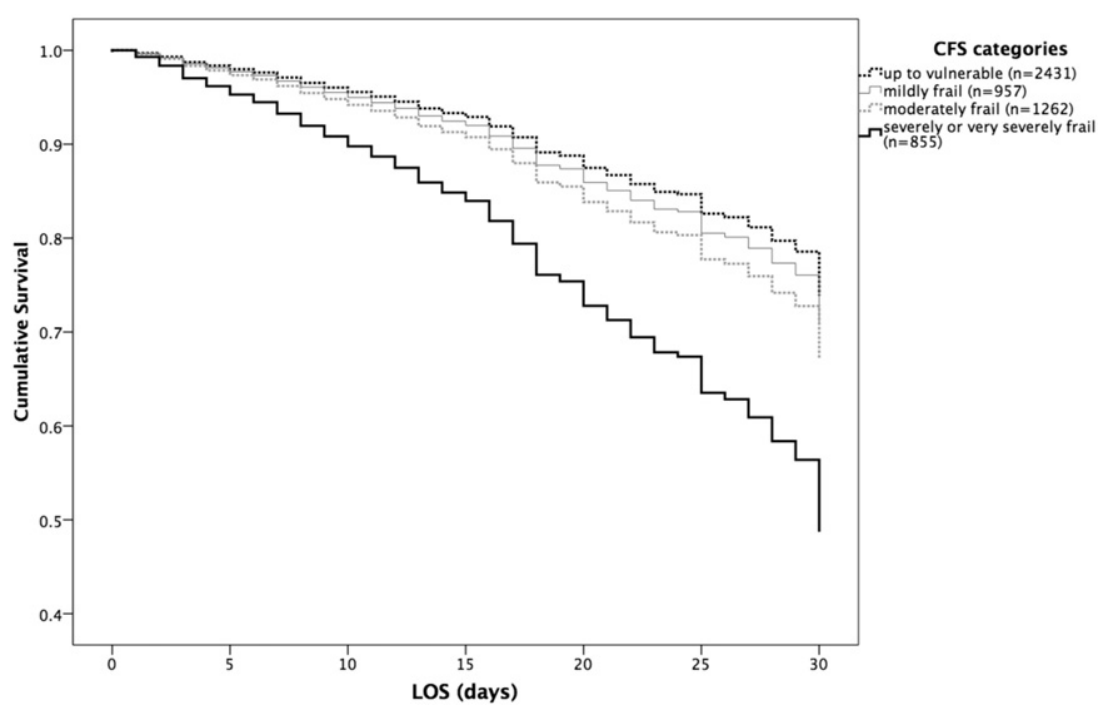

Fig. 2. Age and sex adjusted survival curves for the total sample $(\mathrm{n}=5505)$, by Clinical Frailty Scale (CFS) categories.

Therefore, it was not mandatory for patients whose length of stay was $<72 \mathrm{~h}$ and this may have impacted on practice.

In addition, the significant proportion of missing data may be due to coding issues in the electronic database. It is possible that in some instances, variables such as the ED-MEWS score were measured but not entered in the electronic flowsheet by the attending clinicians. It is unlikely that the ED-MEWS was not measured in the $8 \%$ of patients on whom it was missing on the database. We conducted a pragmatic service evaluation and we have no means of knowing how accurate the coding of the variables used was in real clinical practice. However, undertriage of AIS is recognized in the field of Geriatric Emergency Medicine and apart from non-adherence, reasons for undertriage in older ED patients are various and complex [28,29].

Our results suggested that having history of dementia without a current cognitive concern seemed to be associated with increased survival. A possible reason for this may be that as defined in our database, current cognitive concern (without a history of dementia) may tend to capture delirium, which is often associated with higher AIS [30]. Dementia without delirium is likely to reflect lower AIS, and hence be associated with increased survival and higher LOS [20].

Despite the fact that frailty is an independent predictor of hospitalization [31], our results help understand why frailty-rating scales alone have been of limited use in risk stratifying older people in the acute setting [32]. Indeed, studies with frailty scales in acute settings must take acuity into account. AIS metrics are not usually necessary in community studies where acuity is less likely to confound the association with

Table 2

Results of the Cox proportional hazards regression model. CI: confidence interval. CCI: Charlson Comorbidity Index; CFS: Clinical Frailty Scale; ED-MEWS: Modified Early Warning Score in the Emergency Department.

\begin{tabular}{lllll}
\hline & $\begin{array}{l}\text { Hazard ratio } \\
(\mathrm{HR})\end{array}$ & $\begin{array}{l}\text { 95\% CI for HR } \\
\text { (lower) }\end{array}$ & $\begin{array}{l}\text { 95\% CI for HR } \\
\text { (upper) }\end{array}$ & $\mathrm{p}$ \\
\hline $\begin{array}{l}\text { Age (years) } \\
\text { Female sex }\end{array}$ & 1.04 & 1.02 & 1.07 & $<0.001$ \\
$\begin{array}{l}\text { Discharge by medical } \\
\quad \text { specialty }\end{array}$ & 0.93 & 0.74 & 1.18 & 0.565 \\
CCI & 1.21 & 0.86 & 1.70 & 0.281 \\
History of dementia & 1.08 & 1.05 & 1.11 & $<0.001$ \\
Current cognitive concern & 0.49 & 0.35 & 0.69 & $<0.001$ \\
CFS 1-4 (reference) & - & 0.62 & 1.27 & 0.510 \\
CFS 5 & 1.08 & - & - & $<0.001$ \\
CFS 6 & 1.31 & 0.74 & 1.59 & 0.686 \\
CFS 7-8 & 2.10 & 1.52 & 1.81 & 0.111 \\
ED-MEWS 4 or more & 2.87 & 2.27 & 2.92 & $<0.001$ \\
\hline
\end{tabular}

adverse outcomes. In the community, the identification of severe or very severe frailty (without acuity information) may also have practical implications. Firstly, community interventions may help identify remediable factors and personalize interventions aimed at reversing frailty, which if achieved may predispose patients to better outcomes when acuity strikes. In addition, there is evidence that frail patients with acute organ failure often have high rates of geriatric syndromes at hospital admission but low rates of previous participation in advance care planning activities [33]. Hence, frailty can also be a focus for personalization of care and advance care planning in the community.

\section{Conclusion}

Frailty has the potential to become a powerful instrument in daily clinical practice [34], adding to acuity in the prediction of inpatient mortality in older people, independently of potential confounders. NHS hospitals may find it informative to undertake large scale assessment of frailty in older adults admitted via the emergency pathways. When combined with acute illness severity data, this process may provide hospitals with information which will help define the acute needs of the local population and aid in the development of care pathways for frail adults.

There are several validated instruments for measuring frailty and clinicians should choose one best suited to the clinical environment and/ or patient population they are working with [9]. Future prospective studies are needed to build on the findings presented here and confirm that measurement of frailty in the acute setting can help identify individuals at particular risk of poor hospital outcomes. The gold standard management for acutely hospitalized older adults is the Comprehensive Geriatric Assessment (CGA) approach [35]; and interventional trials are needed to establish whether identification of risk at the front door via routinely collected measures such as frailty and acuity may help target CGA to those who need it the most and enhance patient outcomes.

\section{Disclosure statement}

The authors state that they have no conflicts of interest.

\section{Acknowledgments}

We wish to thank all the members of the acute teams in our hospital, without which this initiative would have not been possible. Licensed access to the hospital's information systems is also gratefully acknowledged. 
Appendix A. ED-MEWS: components, scoring and escalation protocol. HR: heart rate (beats per minute); RR: respiratory rate (per minute); SBP: systolic blood pressure (mmHg); AVPU: Alert, responds to Voice, responds to Pain, Unresponsive; GCS: Glasgow Coma Scale; Temp: body temperature (degrees Celsius); minimum score $=0$ points; maximum score $=15$ points. Escalation trigger (i.e. immediate referral to doctor for clinical review): 4 or more points

\begin{tabular}{|c|c|c|c|c|c|c|c|}
\hline & 3 & 2 & 1 & 0 & 1 & 2 & 3 \\
\hline HR & $<40$ & $41-50$ & $51-60$ & $61-90$ & $91-110$ & $111-129$ & $\geq 130$ \\
\hline $\mathrm{RR}$ & $\leq 6$ & $7-8$ & - & $9-14$ & $15-20$ & $21-29$ & $\geq 30$ \\
\hline SBP & $\leq 70$ & $71-80$ & $81-100$ & $101-180$ & - & $\geq 181$ & - \\
\hline AVPU & U & $\mathrm{P}$ & V & A & & & \\
\hline GCS & & & & 15 & 14 & $9-13$ & $\leq 8$ \\
\hline Temp & - & $<35.0$ & - & $35.0-38.4$ & - & $38.5-39.0$ & $\geq 39.0$ \\
\hline
\end{tabular}

Appendix B. Correlation between the CFS and the ED-MEWS (continuous variables). Two-tailed Spearman's rho correlation coefficient: 0.17 $(\mathrm{p}<0.001, \mathrm{n}=5505)$

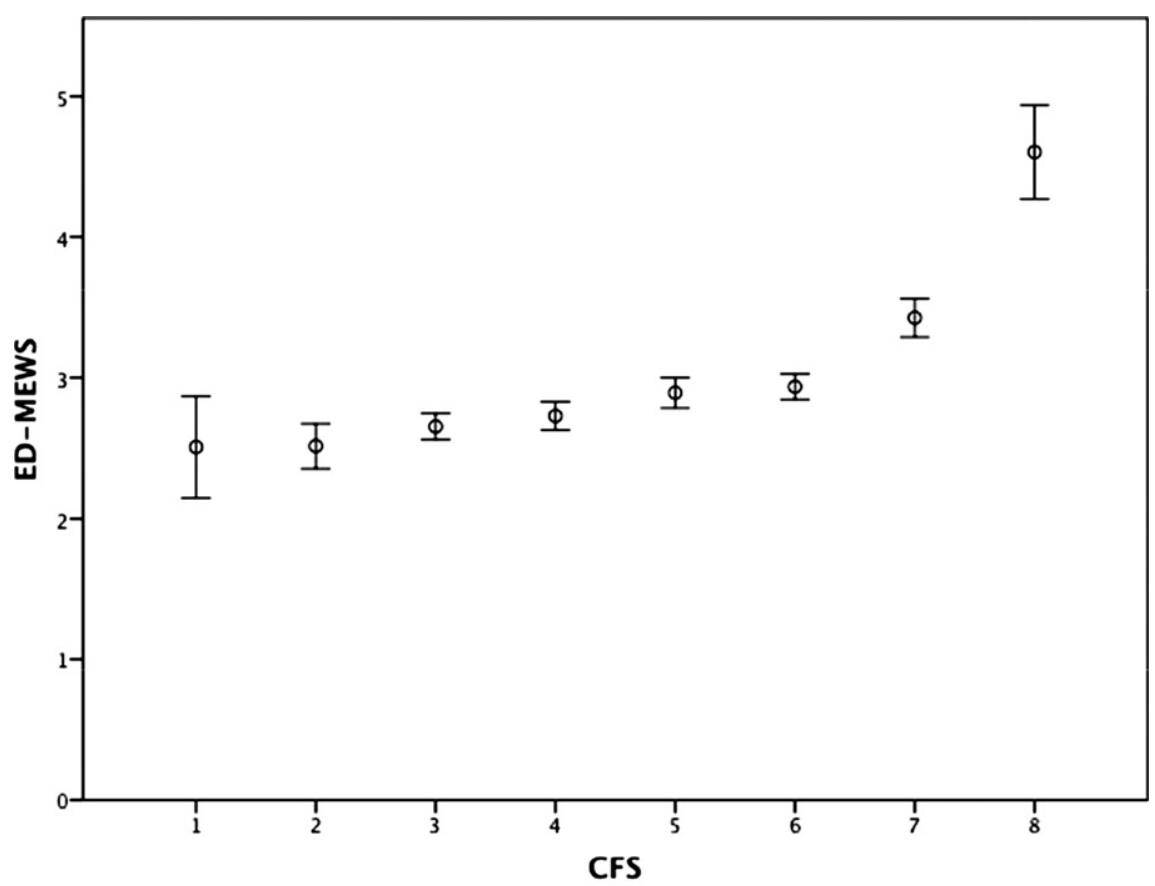

Error Bars: 95\% confidence intervals 
Appendix C. Cumulative hazards functions for the covariates in the Cox regression model
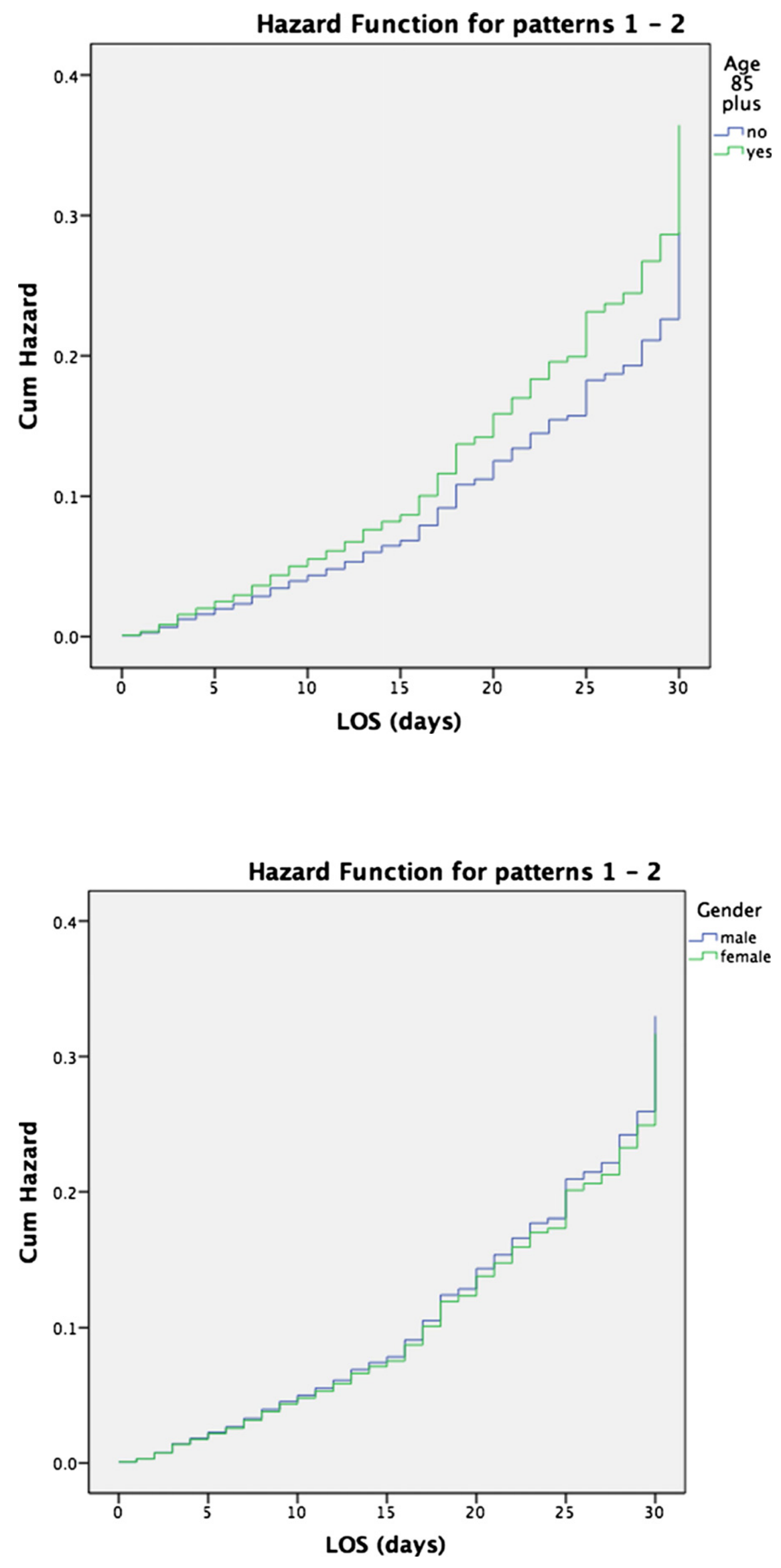

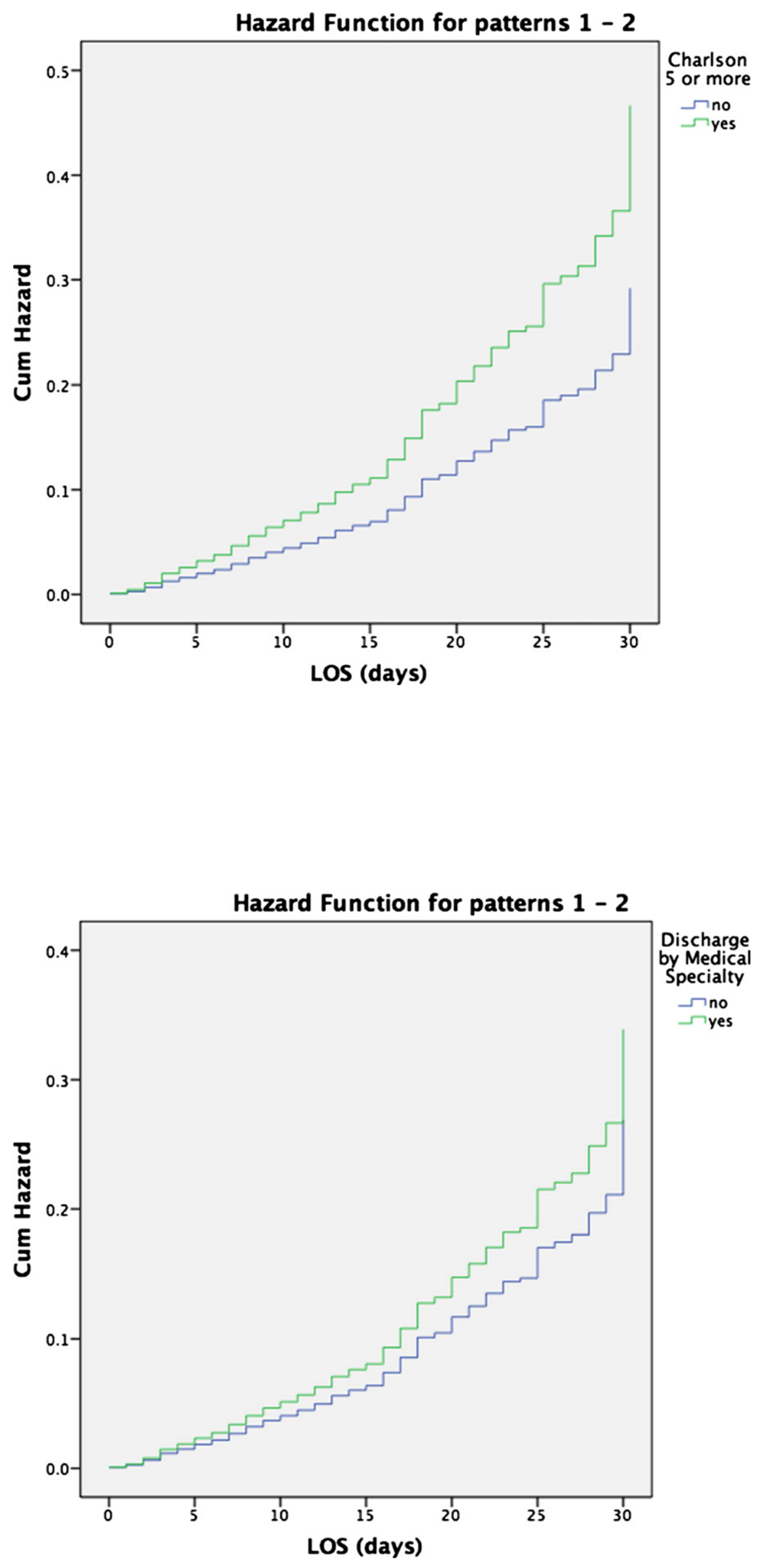

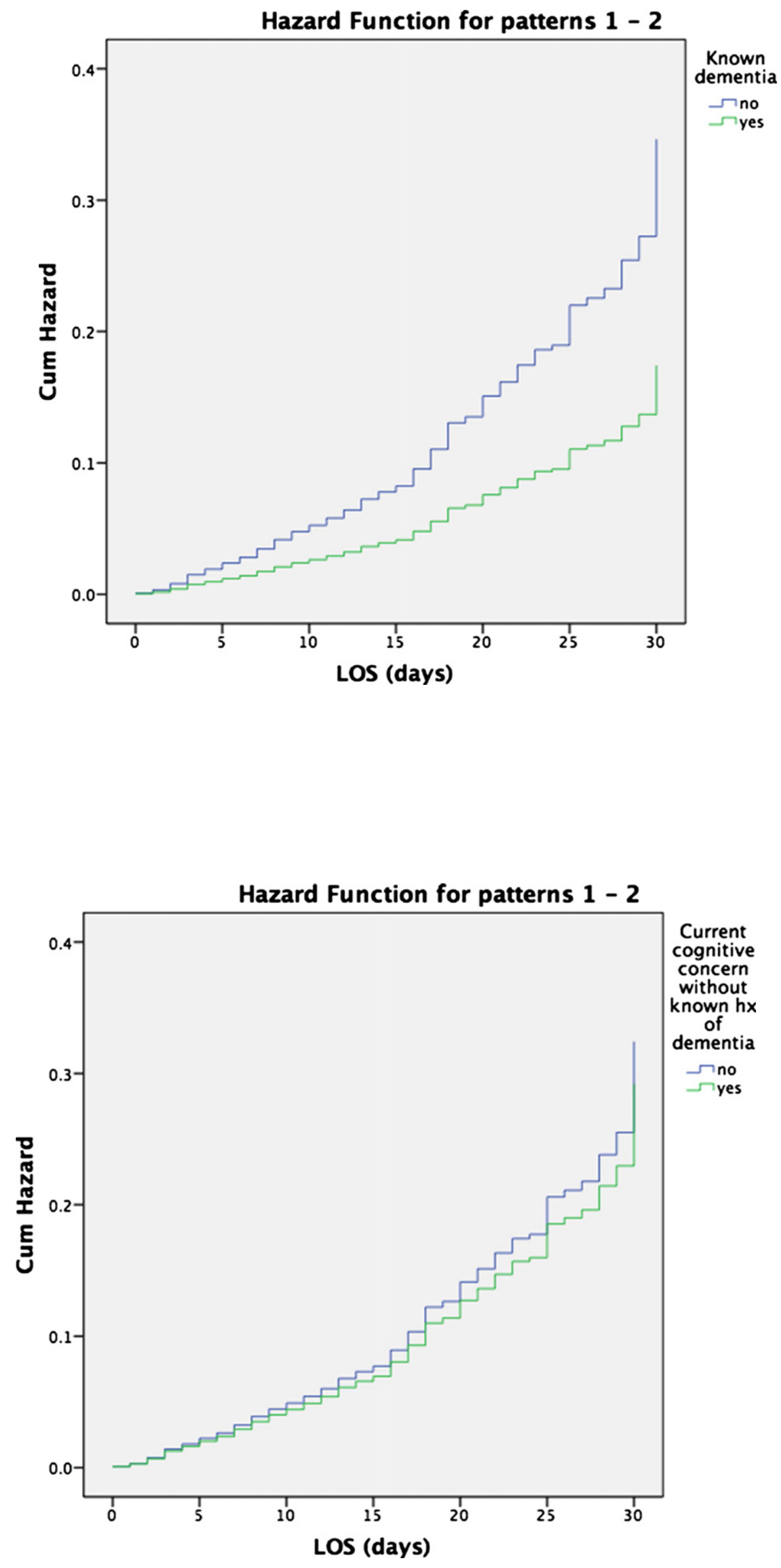

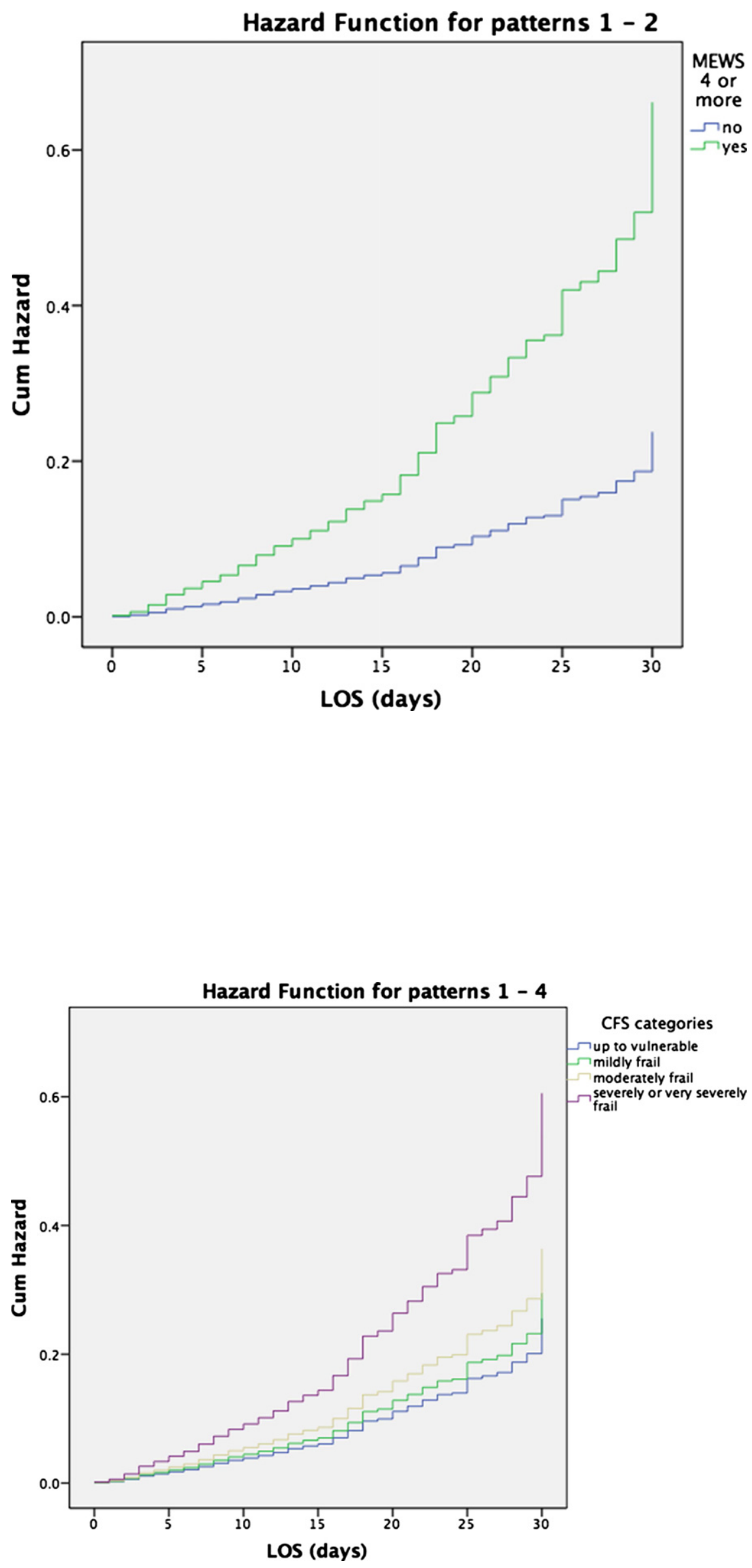


\section{Appendix D. Sensitivity analyses: missing data}

Clinical Frailty Scale (CFS) and ED-MEWS scores were missing in $28.8 \%$ and $8.4 \%$ of patients respectively. Patients with missing data were younger and were more likely to be men, less cognitively impaired, discharged by a non-medical specialty, have a shorter length of stay and to die. Therefore, in order to address any potential bias introduced by the missing data, multiple imputation by chained equations was performed using Stata (version 12.0) to impute missing data in the CFS and ED-MEWS score. Multiple imputation is an efficient and appropriate method to account for missing data [1].

Ordinal logistic regression models were used to impute missing CFS and ED-MEWS scores and the dataset was restricted to 8120 patients who had complete data in all other variables. These were age, history of dementia (yes/no), current cognitive concern (yes/ no), sex, Charlson Comorbidity Index and discharge by a medical specialty (yes/no). These were entered into the imputation models along with the outcome (inpatient death at 30 days), which was entered using two parameters, one indicating the event (dead) and one indicating an estimate of the cumulative hazard since baseline (Nelson-Aalen indicator). All associations to be analyzed in the final analytical model were included in the imputation model. Omitting variables biases associations towards the null and can lead to erroneous conclusions [2,3].

Since nearly $30 \%$ of the CFS scores were missing, 30 imputed datasets were created and Cox proportional hazards regression was run in all 30 imputed datasets. The estimates generated were then combined using Rubin's rules $[4,5]$.

\section{Table}

Cox proportional hazards regression model (with imputed missing data).

\begin{tabular}{|c|c|c|c|c|}
\hline & \multirow[t]{2}{*}{ Hazard ratio } & \multicolumn{2}{|c|}{$\begin{array}{l}\text { 95\% Confidence } \\
\text { intervals }\end{array}$} & \multirow[t]{2}{*}{$\mathrm{p}$ value } \\
\hline & & Lower & Upper & \\
\hline Age (years) & 1.04 & 1.03 & 1.06 & $<0.001$ \\
\hline Female sex & 0.83 & 0.70 & 0.99 & 0.04 \\
\hline Discharge by medical specialty & 0.95 & 0.76 & 1.19 & 0.67 \\
\hline $\mathrm{CCI}$ & 1.06 & 1.04 & 1.09 & $<0.001$ \\
\hline History of Dementia & 0.38 & 0.29 & 0.51 & $<0.001$ \\
\hline Current cognitive concern & 0.52 & 0.38 & 0.71 & $<0.001$ \\
\hline \multicolumn{5}{|l|}{ CFS } \\
\hline 1-4 (up to vulnerable) & Ref & & & \\
\hline 5 (mildly frail) & 1.17 & 0.84 & 1.61 & 0.35 \\
\hline 6 (moderately frail) & 1.46 & 1.09 & 1.96 & 0.01 \\
\hline 7-8 (severely/very sev. frail) & 1.98 & 1.47 & 2.66 & $<0.001$ \\
\hline ED-MEWS 4 or more & 3.44 & 2.83 & 4.18 & $<0.001$ \\
\hline
\end{tabular}

$\mathrm{N}=8120$

References for Appendix D:

[1]. Sterne JAC, White IR, Carlin JB, Spratt M, Royston P, Kenward MG, et al. Multiple imputation for missing data in epidemiological and clinical research: potential and pitfalls. BMJ. 2009 Jan.;338:b2393.

[2]. Hippisley-Cox J, Coupland C, Vinogradova Y, Robson J, May M, Brindle P. Derivation and validation of QRISK, a new cardiovascular disease risk score for the United Kingdom: prospective open cohort study. BMJ. 2007 Jul. 21;335(7611):136.

[3]. Moons KGM, Donders R a RT, Stijnen T, Harrell FE. Using the outcome for imputation of missing predictor values was preferred. J Clin Epidemiol. 2006 Oct.;59(10):1092-101.

[4]. Rubin D. Multiple imputation for nonresponse in surveys. New York: John Wiley and Sons; 2004.

[5]. Marshall A, Altman DG, Holder RL, Royston P. Combining estimates of interest in prognostic modeling studies after multiple imputation: current practice and guidelines. BMC Med Res Methodol. 2009 Jan.;9:57.

\section{References}

[1] Subbe CP, Kruger M, Rutherford P, Gemmel L. Validation of a modified Early Warning Score in medical admissions. QJM 2001;94(10):521-6.

[2] Smith GB, Prytherch DR, Meredith P, Schmidt PE. Early warning scores: unravelling detection and escalation. Int J Health Care Qual Assur 2015;28(8):872-5.

[3] Alam N, Hobbelink EL, van Tienhoven AJ, van de Ven PM, Jansma EP, Nanayakkara PW. The impact of the use of the Early Warning Score (EWS) on patient outcomes: a systematic review. Resuscitation 2014;85(5):587-94.

[4] Chotirmall SH, Picardo S, Lyons J, D'Alton M, O'Riordan D, Silke B. Disabling disease codes predict worse outcomes for acute medical admissions. Intern Med J 2014; 44(6):546-53.

[5] Soong J, Poots AJ, Scott S, et al. Quantifying the prevalence of frailty in English hospitals. BMJ Open 2015;5(10), e008456.

[6] Clegg A, Young J, Iliffe S, Rikkert MO, Rockwood K. Frailty in elderly people. Lancet 2013;381(9868):752-62.

[7] Romero-Ortuno R, O'Shea D. Fitness and frailty: opposite ends of a challenging continuum! Will the end of age discrimination make frailty assessments an imperative? Age Ageing 2013;42(3):279-80.

[8] Lloyd-Sherlock P, McKee M, Ebrahim S, et al. Population ageing and health. Lancet 2012;379(9823):1295-6.

[9] Dent E, Kowal P, Hoogendijk EO. Frailty measurement in research and clinical practice: a review. Eur J Intern Med 2016;31:3-10.

[10] Martin FC, Brighton P. Frailty: different tools for different purposes? Age Ageing 2008;37(2):129-31.

[11] Cesari M, Gambassi G, van Kan GA, Vellas B. The frailty phenotype and the frailty index: different instruments for different purposes. Age Ageing 2014:43(1):10-2.

[12] Illsley A, Clegg A. Assessment of frailty in the inpatient setting. Br J Hosp Med (Lond) 2016;77(1):29-32.

[13] Wyrko Z. Frailty at the front door. Clin Med (Lond) 2015;15(4):377-81.

[14] Conroy S, Chikura G. Emergency care for frail older people-urgent AND importantbut what works? Age Ageing 2015;44(5):724-5.

[15] Dent E, Hoogendijk EO, Cardona-Morrell M, Hillman K. Frailty in emergency departments. Lancet 2016;387(10017):434.

[16] Soong J, Poots AJ, Scott S, Donald K, Bell D. Developing and validating a risk prediction model for acute care based on frailty syndromes. BMJ Open 2015;5(10), e008457.

[17] Charlson ME, Pompei P, Ales KL, MacKenzie CR. A new method of classifying prognostic comorbidity in longitudinal studies: development and validation. J Chronic Dis 1987;40(5):373-83.

[18] Rockwood K, Song X, MacKnight C, et al. A global clinical measure of fitness and frailty in elderly people. CMAJ 2005;173(5):489-95.

[19] Subbe CP, Burford C, Jeune IL, Masterton-Smith C, Ward D. Relationship between input and output in acute medicine - secondary analysis of the Society for Acute Medicine's benchmarking audit 2013 (SAMBA '13). Clin Med 2015;15(1):15-9.

[20] Wallis SJ, Wall J, Biram RW, Romero-Ortuno R. Association of the clinical frailty scale with hospital outcomes. QJM 2015;108(12):943-9.

[21] Greiner M, Pfeiffer D, Smith RD. Principles and practical application of the receiveroperating characteristic analysis for diagnostic tests. Prev Vet Med 2000;45(1-2):23-41.

[22] Thompson D, Rumley-Buss M, Conroy S. Transforming emergency services for frail older people in hospital. Nurs Manag (Harrow) 2015;22(3):18-9.

[23] Cournane S, Byrne D, O'Riordan D, Fitzgerald B, Silke B. Chronic disabling disease-impact on outcomes and costs in emergency medical admissions. QJM 2015;108(5):387-96.

[24] Maher JM, Markey JC, Ebert-May D. The other half of the story: effect size analysis in quantitative research. CBE Life Sci Educ 2013;12(3):345-51.

[25] Romero-Ortuno R, O'Shea D, Silke B. Predicting the in-patient outcomes of acute medical admissions from the nursing home: the experience of St James's Hospital, Dublin, 2002-2010. Geriatr Gerontol Int 2012;12(4):703-13.

[26] Wittenberg R, Sharpin L, McCormick B, Hurst J. Understanding emergency hospital admissions of older people. Centre for Health Service Economics \& Organisation (CHSEO) 2014; report no. 6. Available online: http://www.chseo.org.uk/papers.html.

[27] Boyle AA, Ahmed V, Palmer CR, Bennett TJ, Robinson SM. Reductions in hospital admissions and mortality rates observed after integrating emergency care: a natural experiment. BMJ Open 2012:2(4).

[28] Grossmann FF, Zumbrunn T, Ciprian S, et al. Undertriage in older emergency department patients-tilting against windmills? PLoS One 2014:9(8), e106203.

[29] Grossmann FF, Zumbrunn T, Frauchiger A, Delport K, Bingisser R, Nickel CH. At risk of undertriage? Testing the performance and accuracy of the emergency severity index in older emergency department patients. Ann Emerg Med 2012;60(3):317-25 e3.

[30] Ahmed S, Leurent B, Sampson EL. Risk factors for incident delirium among older people in acute hospital medical units: a systematic review and meta-analysis. Age Ageing 2014;43(3):326-33.

[31] Kojima G. Frailty as a predictor of hospitalisation among community-dwelling older people: a systematic review and meta-analysis. J Epidemiol Community Health 2016

[32] Wou F, Gladman JR, Bradshaw L, Franklin M, Edmans J, Conroy SP. The predictive properties of frailty-rating scales in the acute medical unit. Age Ageing 2013; 42(6):776-81.

[33] Huijberts S, Buurman BM, de Rooij SE. End-of-life care during and after an acute hospitalization in older patients with cancer, end-stage organ failure, or frailty: a subanalysis of a prospective cohort study. Palliat Med 2016;30(1):75-82.

[34] Rodriguez-Manas L, Fried LP. Frailty in the clinical scenario. Lancet 2015;385(9968): e7-9.

[35] Ellis G, Whitehead MA, O'Neill D, Langhorne P, Robinson D. Comprehensive geriatric assessment for older adults admitted to hospital. Cochrane Database Syst Rev 2011; 7, CD006211. 